\title{
Time-reversal symmetry violation in molecules induced by nuclear magnetic quadrupole moments
}

\author{
V. V. Flambaum ${ }^{1}$, D. DeMille ${ }^{2}$, and M. G. Kozlov ${ }^{3,4}$ \\ ${ }^{1}$ School of Physics, The University of New South Wales, Sydney NSW 2052, Australia \\ ${ }^{2}$ Department of Physics, P.O. Box 208120, Yale University, New Haven, Connecticut 06520, USA \\ ${ }^{3}$ Petersburg Nuclear Physics Institute, Gatchina 188300, Russia and \\ ${ }^{4}$ St. Petersburg Electrotechnical University "LETI", Prof. Popov Str. 5, 197376 St. Petersburg
}

(Dated: October 12, 2018)

\begin{abstract}
Recent measurements in paramagnetic molecules improved the limit on the electron electric dipole moment (EDM) by an order of magnitude. Time-reversal $(\mathrm{T})$ and parity $(\mathrm{P})$ symmetry violation in molecules may also come from their nuclei. We point out that nuclear T,P-odd effects are amplified in paramagnetic molecules containing deformed nuclei, where the primary effects arise from the T,P-odd nuclear magnetic quadrupole moment (MQM). We perform calculations of T,P-odd effects in the molecules $\mathrm{TaN}, \mathrm{ThO}, \mathrm{ThF}^{+}, \mathrm{HfF}^{+}, \mathrm{YbF}, \mathrm{HgF}$, and $\mathrm{BaF}$ induced by MQMs. We compare our results with those for the diamagnetic TIF molecule, where the T,P-odd effects are produced by the nuclear Schiff moment. We argue that measurements in molecules with MQMs may provide improved limits on the strength of T,P-odd nuclear forces, on the proton, neutron and quark EDMs, on quark chromo-EDMs, and on the QCD $\theta$-term and CP-violating quark interactions.
\end{abstract}

In order to explain the matter-antimatter asymmetry in the universe, additional sources of CP-violation (or equivalently T-violation, assuming CPT symmetry) are required, beyond those in the Standard Model of particle physics [1]. Measurements of T,P-violating electric dipole moments (EDMs) are an efficient way to search for this type of new physics [2]. For example, the parameter space for $\mathrm{CP}$-violation in supersymmetric theories is very strongly limited by EDM measurements [3, 4].

Measurements of nuclear T,P-odd effects have focused on heavy diamagnetic atoms [5 8] and molecules [9]. In these systems, the EDM of the nucleus is entirely screened by electrons (the Schiff theorem [10]) and contributes negligibly to the measurable EDM. Instead, here the observable EDM is generated by the nuclear Schiff moment (SM). The SM is an intra-nuclear charge distribution, generated by T,P-odd interactions within the nucleus, which can induce an atomic/molecular EDM by polarizing the bound electrons [11, 12]. The SM has size $\sim r_{N}^{2} d_{N}$ where $r_{N}\left(d_{N}\right)$ is the nuclear radius (EDM). Since $r_{N}$ is very small compared to the electron orbital size, the atomic EDM produced by the nuclear SM is much smaller than $d_{N}$. By contrast, the magnetic interaction between nuclear moments and electrons is not screened. The lowest T,P-odd magnetic moment is the magnetic quadrupole moment (MQM). It was shown in 11] that in paramagnetic atoms and molecules, the nuclear MQM produces a larger EDM than does the SM (see also [13, 14]), for the same underlying sources of $\mathrm{CP}$-violation. Moreover, the MQM has a collective nature and is significantly enhanced in deformed nuclei (like the ordinary electric quadrupole moment) [15].

Unfortunately, it has been difficult to devise experiments sensitive to MQMs, and hence to take advantage of these mechanisms for enhanced effects of T,P-odd hadronic physics. There are several problems [2]. Use of paramagnetic rather than diamagnetic systems generally leads to much shorter spin coherence times and hence drastically reduced energy resolution. Susceptibility to magnetic noise is also greatly increased in paramagnetic systems.

Recently, however, there have been experimental advances that could be used to exploit the intrinsic advantages of MQMs. In particular, it has become possible to perform EDM measurements using molecules in paramagnetic ${ }^{3} \Delta_{1}$ electronic states. Due to a cancellation of electronic orbital and spin magnetic moments their net magnetic moment is on the order of a nuclear magneton 16]. They also have $\Omega$-doublet substructure, which both allows full polarization in modest external electric fields and provides a means to cancel many systematic errors 17, 18. Intense, slow molecular beams [19, 20] and techniques for spin-precession measurements both on such beams 21] and on trapped molecular ions 22] have been developed. Using these methods, recently the limit on the electron EDM (eEDM) was improved by an order of magnitude using the ${ }^{3} \Delta_{1}$ state of ${ }^{232} \mathrm{ThO}$ [23]; substantial further improvements in sensitivity are anticipated 22, 23].

In this paper, we point out the possibility to use this type of molecular state to search for T,P-odd interactions in the hadronic sector. This approach takes advantage of the dramatically enhanced energy shifts associated with the strong electric polarization of molecules, as was exploited in older experiments searching for the $\mathrm{SM}$ of ${ }^{205} \mathrm{Tl}$ in $\mathrm{TlF}$ [9]. However, it also uses the enhanced effects of the MQM, especially in deformed nuclei, to further boost the sensitivity relative to experiments using atomic ${ }^{199} \mathrm{Hg}$, where measurements of the SM now place the strongest limits on most underlying effects [5].

The MQM also appears more amenable to a reliable interpretation than the SM, due to the differences in nu- 
clear structure that give rise to these effects. In the expression for the SM there are two terms that have close values and opposite sign 11. This makes the result sensitive to corrections such as those due to finite nuclear size 24] and many-body effects 25 27]. Also, in EDM experiments using nuclei with a valence neutron (e.g. ${ }^{199} \mathrm{Hg}$ ), the direct valence nucleon contribution is zero and the $\mathrm{SM}$ is generated primarily by polarization of the nuclear core by its T,P-odd interaction with the valence neutron [25]. This makes calculations of the SM especially sensitive to many-body corrections, which significantly suppress the final results and make them unstable [25 27]. For the MQM a valence nucleon gives the main contribution, so the result should be less sensitive to many-body corrections (the T,P-odd core polarization contribution to the MQM was estimated in 28]).

The eEDM, SM, and MQM contributions to the T,Podd effects in paramagnetic diatomic molecules are described by the effective molecular Hamiltonian [11]:

$$
H=W_{d} d_{e} \boldsymbol{S} \cdot \boldsymbol{n}+W_{Q} \frac{Q}{I} \boldsymbol{I} \cdot \boldsymbol{n}-\frac{W_{M} M}{2 I(2 I-1)} \boldsymbol{S} \hat{\boldsymbol{T}} \boldsymbol{n} .
$$

Here $\boldsymbol{n}$ is a unit vector along the molecular axis, $\boldsymbol{I}$ is the nuclear spin, $\boldsymbol{S}$ is the effective electronic spin, $d_{e}$ is the eEDM, $Q$ is the nuclear SM, and $M$ is the nuclear MQM, with components

$$
M_{i, k}=3 M /[2 I(2 I-1)] T_{i, k},
$$

where $T_{i, k}=I_{i} I_{k}+I_{k} I_{i}-\frac{2}{3} \delta_{i, k} I(I+1)$. For the maximal nuclear spin projection $I_{z}=I$ along $\boldsymbol{n}$, we have $M_{z z}=M$ and the MQM energy shift in Eq. (11) is $-\frac{1}{3} W_{M} M S_{z}$. This shift is quadrupled by switching the directions of the external fields [29]. The value of $S$ is defined as $S=|\Omega|$, where $\Omega=\boldsymbol{J}_{e} \cdot \boldsymbol{n}$ is the projection of the total electronic angular momentum $\boldsymbol{J}_{e}$ on the molecular axis. The parameters $W_{d}, W_{Q}$ and $W_{M}$ must be found from molecular electronic structure calculations; some useful equations for them are presented in [11].

Nuclear calculations of MQM. The MQM of a nucleus can arise both due to the EDMs of the constituent nucleons, and due to intra-nuclear T,P-odd forces. The calculation of the MQM produced by a valence nucleon EDM was done in Ref. [30]. The MQM produced by T,Podd nuclear forces was calculated in [11] (see also [31]). It is important that T,P-odd nuclear forces produce T,Podd nuclear moments 1-2 orders of magnitude larger than those caused by nucleon EDMs [1]]. Following [11, 30] we can present the MQM of a valence nucleon as

$$
\begin{aligned}
M & =M_{0}^{v}(2 I-1) t_{I}, \\
M_{0}^{v} & =\left[d_{v}+\xi_{v}\left(\mu_{v}-q_{v}\right)\right] \hbar /\left(m_{p} c\right),
\end{aligned}
$$

where $t_{I}=1$ for $I=l+\frac{1}{2}$ and $t_{I}=-\frac{I}{I+1}$ for $I=l-\frac{1}{2}, I$ and $l$ are the total and orbital angular momenta of the valence nucleon denoted by $v=p, n ; d_{v}$ is the valence nucleon
EDM, $\xi_{v}=-2 \cdot 10^{-21} \eta_{v}(e \cdot \mathrm{cm}), \eta_{v}$ is the dimensionless strength constant of the T,P-odd nuclear potential $H_{T, P}=\eta_{v} G_{F} /\left(2^{3 / 2} m_{p}\right)(\boldsymbol{\sigma} \cdot \nabla \rho), \rho$ is the total nucleon number density, $G_{F}$ is the Fermi constant, $m_{p}$ is the proton mass, and the nucleon magnetic moments and charges are $\mu_{p}=2.79, q_{p}=1$ and $\mu_{n}=-1.91, q_{n}=0$.

The T,P-odd nuclear forces are dominated by $\pi_{0}$ meson exchange. Therefore, we may express the strength constants via the strong $\pi N N$ coupling constant $g=13.6$ and three T,P-odd $\pi N N$ coupling constants $\bar{g}_{T}$ corresponding to the isospin channels $T=0,1,2: \eta_{n}=-\eta_{p} \approx$ $5 \cdot 10^{6} g\left(\bar{g}_{1}+0.4 \bar{g}_{2}-0.2 \bar{g}_{0}\right)$. The numerical coefficient comes from $\left[G_{F} m_{\pi}^{2} / 2^{1 / 2}\right]^{-1}=6.7 \cdot 10^{6}$ times the factor 0.7 corresponding to the zero range reduction of the finite range interaction due to the $\pi_{0}$-exchange [11, 32]. As a result, we obtain

$$
\begin{aligned}
M_{0}^{v}(g) & =\left[g\left(\bar{g}_{1}+0.4 \bar{g}_{2}-0.2 \bar{g}_{0}\right)\right. \\
& \left.+d_{v} /\left(1.4 \cdot 10^{-14} e \cdot \mathrm{cm}\right)\right] \cdot 3 \cdot 10^{-28} e \cdot \mathrm{cm}^{2} .
\end{aligned}
$$

In the numerical coefficient here we included two additional correction factors. First, more accurate numerical calculations in a Saxon-Woods potential [1, 32] give larger values of MQM (by a factor $\sim 1.2$ ) than the simple analytical solution in Eq. (4). Second, many-body corrections reduce the effective strength constants $\eta_{v}$ of the $\mathrm{T}, \mathrm{P}$-odd potential by $\sim 1.5$ times $[15,33]$.

Finally, we can use previously derived relations between underlying sources of CP-violation and the nuclear T,P-odd forces, to express the MQM in terms of these more fundamental quantities. For example, the QCD CP violation parameter $\tilde{\theta}$ induces a nuclear T,P-odd force described by the relation $g \bar{g}_{0}=-0.37 \tilde{\theta}[34$, leading to a valence nucleon MQM:

$$
M_{0}^{p}(\theta) \approx M_{0}^{n}(\theta) \approx 2 \cdot 10^{-29} \tilde{\theta} e \cdot \mathrm{cm}^{2} .
$$

Contributions of $\tilde{\theta}$ to the MQM via the EDMs of the neu$\operatorname{tron}\left(d_{n}=1.2 \cdot 10^{-16} \tilde{\theta} e \cdot \mathrm{cm}[35]\right)$ and proton $\left(d_{p} \approx-d_{n}\right)$ are an order of magnitude smaller. Note that the valence contributions of $\bar{g}_{0}$ and $\tilde{\theta}$ to the MQM are suppressed by the small factor $(N-Z) / A \approx 0.2$, where $N$ and $Z$ are the neutron and proton numbers and $A=N+Z$. The contribution of the T,P-odd core polarization 28] has no such suppression and may increase the value of MQM in terms of $\bar{g}_{0}$ and $\tilde{\theta}$.

Similarly, we can express MQM in terms of the $u$ and $d$ quark EDMs $d_{u, d}$ and chromo-EDMs $\tilde{d}_{u, d}$ using the relations $g \bar{g}_{1}=4 \cdot 10^{15}\left(\tilde{d}_{u}-\tilde{d}_{d}\right) / \mathrm{cm}, g \bar{g}_{0}=0.8 \cdot 10^{15}\left(\tilde{d}_{u}+\right.$ $\left.\tilde{d}_{n}\right) / \mathrm{cm}, d_{p}=1.1 e\left(\tilde{d}_{u}+0.5 \tilde{d}_{d}\right)+1.4 d_{u}+0.35 d_{d}, d_{n}=$ $1.1 e\left(\tilde{d}_{d}+0.5 \tilde{d}_{u}\right)+1.4 d_{d}+0.35 d_{u}[\underline{3}$. We find finally

$$
M_{0}^{p}(\tilde{d}) \approx M_{0}^{n}(\tilde{d}) \approx 1.2 \cdot 10^{-12}\left(\tilde{d}_{u}-\tilde{d}_{d}\right) \cdot e \cdot \mathrm{cm} .
$$

Note that the contributions of $d_{n}$ and $d_{p}$ to this expression are only a few percent and are neglected. 
TABLE I: Nuclear MQMs $M$ derived from Eqs. (2131819) and the orbital occupation numbers given in 36]. The values of $M_{0}^{p}$ and $M_{0}^{n}$ in terms of different constants of CP-violating interactions and EDM are given in Eqs. (4- 7). The values for the spherical nuclei ${ }^{137} \mathrm{Ba}$ and ${ }^{201} \mathrm{Hg}$ have been presented for comparison; note the typical factor of $10-20$ enhancement for the deformed nuclei.

\begin{tabular}{||c|c||c|c||}
\hline Nucleus & $M$ & Nucleus & $M$ \\
\hline${ }^{181} \mathrm{Ta}$ & $-14 M_{0}^{p}-11 M_{0}^{n}$ & ${ }^{229} \mathrm{Th}$ & $0 M_{0}^{p}-19 M_{0}^{n}$ \\
${ }^{173} \mathrm{Yb}$ & $-10 M_{0}^{p}-10 M_{0}^{n}$ & ${ }^{177} \mathrm{Hf}$ & $-19 M_{0}^{p}-14 M_{0}^{n}$ \\
${ }^{179} \mathrm{Hf}$ & $-13 M_{0}^{p}-13 M_{0}^{n}$ & ${ }^{137} \mathrm{Ba}$ & $0 M_{0}^{p}-1.2 M_{0}^{n}$ \\
${ }^{201} \mathrm{Hg}$ & $0 M_{0}^{p}+2 M_{0}^{n}$ & & \\
\hline
\end{tabular}

For spherical nuclei, the quantum numbers needed to find the valence nucleon contribution to the nuclear MQM are related to the nuclear spin $I$ and parity $P$. For example, the nucleus ${ }^{201} \mathrm{Hg}$ has $I^{P}=\frac{3}{2}^{-}$, with one valence neutron in a $p_{3 / 2}$ state, $I=l+\frac{1}{2}, t_{I}=1$, and $M=2 M_{0}^{n}$.

The situation is more complicated in deformed nuclei, where the MQM has a collective nature. Here, about $A^{2 / 3}$ nucleons belong to open shells due to the shell splitting by the strong quadrupole field. The MQM of a deformed nucleus in the "frozen" frame (rotating together with the nucleus), $M_{z z}^{\text {nucl }}$, is given by [15]:

$$
M_{z z}^{\text {nucl }}=\sum M_{z z}^{\text {single }}\left(I, I_{z}, l\right) n\left(I, I_{z}, l\right),
$$

where $M_{z z}^{\text {single }}\left(I, I_{z}, l\right)$ is given by Eqs. (36) and (2) and $n\left(I, I_{z}, l\right)$ are the single-nucleon orbital occupation numbers, which may be found in Ref. [36]. The MQM in the laboratory frame $M \equiv M^{\text {lab }}$ can be expressed via the MQM in the rotating frame:

$$
M^{\mathrm{lab}}=\frac{I_{t}\left(2 I_{t}-1\right)}{\left(I_{t}+1\right)\left(2 I_{t}+3\right)} M_{z z}^{\mathrm{nucl}},
$$

where $I_{t}$ is the total nuclear spin. Values for the MQMs of various nuclei are given in Table \

Calculations of the MQM effects in molecules. The first estimates of the effects of MQM in many heavy molecules were performed in Ref. 11]. Calculations of the constant $W_{M}$ in Eq. (11) for $\mathrm{BaF}, \mathrm{YbF}$, and $\mathrm{HgF}$ were done in Refs. 37, 38] using a semiempirical approach based on measured molecular hyperfine structure constants.

The parameters $W_{d}$ and $W_{M}$ depend on the molecular wave function in the vicinity of the heavy nucleus, where it can be expanded in partial waves. Up to normalization factors, at short distances these partial waves resemble valence atomic orbitals of the heavy atom. The dominant matrix element for $W_{d}$ is between $s_{1 / 2}$ and $p_{1 / 2}$ waves. The electronic operator for the MQM interaction has higher tensor rank and the dominant matrix element for $W_{M}$ is between $s_{1 / 2}$ and $p_{3 / 2}$ waves. For the $\sigma_{1 / 2}$ orbital at large distances from the nucleus the waves $p_{1 / 2}$ and $p_{3 / 2}$ must combine into a non-relativistic $p_{z}$ wave, which has the form: $\left|p_{z}, \omega\right\rangle=-\frac{2 \omega}{\sqrt{3}}\left|p_{1 / 2}, \omega\right\rangle+\sqrt{\frac{2}{3}}\left|p_{3 / 2}, \omega\right\rangle$, where $\omega= \pm \frac{1}{2}$ is projection of the total angular momentum $\boldsymbol{j}_{e}$ along $\boldsymbol{n}$ (for a many-electron molecular state, $\left.\sum_{i} \omega_{i}=\Omega\right)$. This equation links the amplitudes of the relativistic partial waves $p_{1 / 2}$ and $p_{3 / 2}$. Because of this, the amplitudes in the dominant matrix elements for $W_{d}$ and $W_{M}$ are also linked. Consequently, to first approximation the ratio of $W_{M}$ and $W_{d}$ depends on the nuclear charge $Z$ only:

$$
W_{M}=\frac{9 R_{M}(Z)}{20 r_{0} \alpha Z R_{d}(Z)} W_{d},
$$

where $R_{M}(Z)$ and $R_{d}(Z)$ are the relativistic factors for MQM and eEDM presented in [11, 39] and $r_{0}$ is Bohr radius. This expression holds to $20 \%$ accuracy for the molecules $\mathrm{BaF}, \mathrm{YbF}$, and $\mathrm{HgF}$, where $W_{d}$ and $W_{M}$ were calculated in 37, 38.

Metastable ${ }^{3} \Delta_{1}$ state of the molecules ThO, TaN and ions $\mathrm{HfF}^{+}, \mathrm{ThF}^{+}$. The EDM parameter $W_{d}$ was calculated for the molecule ThO 40, 41] and for the ions $\mathrm{HfF}^{+}$[42 44] and $\mathrm{ThF}^{+}$[40, 45]. We use these results and relation (10) to estimate parameter $W_{M}$ for these systems (see Table II). On the Dirac-Fock level relation (10) holds nicely for atomic ions $\mathrm{Hf}^{+}$and $\mathrm{Th}^{+}$and we expect these estimates of $W_{M}$ to be accurate to about $30 \%$.

There are no calculations of $W_{d}$ for TaN. The electronic state ${ }^{3} \Delta_{1}$ was studied theoretically and experimentally in Ref. [46] and was found to include two uncoupled electrons in $\sigma$ and $\delta$ orbitals. This makes it similar to the ${ }^{3} \Delta_{1}$ state of the molecule ThO. However, here calculations indicate that the $\sigma$ orbital is primarily a mixture of the $6 s$ and $5 d$ orbitals of the heavy atom (Ta), with no admixture of the $p$ wave reported in [46]. The closest analogue to $\mathrm{TaN}$ is $\mathrm{YbF}$. Because of the larger $Z$ and larger binding energies, the atomic MQM matrix element for Ta is 1.6 times bigger than for $\mathrm{Yb}$. On the other hand, the large admixture of the $d$ wave rather than the $p$ wave should lead to smaller molecular matrix elements. Thus, as a very rough estimate for $\mathrm{TaN}$ we take the value of $W_{M}$ from Ref. [38] for $\mathrm{YbF}$ and divide it by 2 to account for the difference in $\Omega$.

In Table Wwe summarize our results for molecules that are used, or considered for EDM experiments. One of the best limits on the eEDM comes from measurements on YbF molecule in ${ }^{2} \Sigma_{1 / 2}$ state [29]. Hence we include calculations of MQM shifts in three such species, which were calculated in [38]. We express the shifts in terms of the fundamental underlying CP-violating physical quantities $d_{p}, \tilde{\theta}$, and $\tilde{d}_{u, d}$. The current limits on these quantities are given in Ref. [5]: $\left|d_{p}\right|<8.6 \cdot 10^{-25} e \cdot \mathrm{cm},|\tilde{\theta}|<2.4 \cdot 10^{-10}$, and $\left|\tilde{d}_{u}-\tilde{d}_{d}\right|<6 \cdot 10^{-27} \mathrm{~cm}$. The values of the frequency shifts produced by the nuclear MQMs are sufficiently large to compete in the improvement of limits on the proton $\mathrm{EDM} d_{p}$, on the $\tilde{\theta}$-term, and on the difference 
TABLE II: Parameter $W_{M}$ and the product $\left|W_{M} M S\right|$ for the heavy nucleus with total nuclear spin $I_{t}$, for molecular states of interest. The last three columns give values of $\left|W_{M} M S\right|$ produced by the proton EDM $d_{p}$, the QCD $\tilde{\theta}$-term, and the difference of the quark chromo-EDMs $\left(\tilde{d}_{u}-\tilde{d}_{d}\right)$.

\begin{tabular}{ccccccc}
\hline Molecule & $I_{t}$ State & $\left|W_{M}\right|$ & \multicolumn{3}{c}{$\left|W_{M} M S\right|(\mu \mathrm{Hz})$} \\
& & $\frac{10^{33} \mathrm{~Hz}}{\mathrm{ecm}^{2}}$ & $\frac{10^{25} d_{p}}{e \cdot \mathrm{cm}}$ & $10^{10} \tilde{\theta}$ & $\frac{10^{27}\left(\tilde{d}_{u}-\tilde{d}_{d}\right)}{\mathrm{cm}}$ \\
\hline${ }^{135,137} \mathrm{BaF}$ & $\frac{3}{2}$ & ${ }^{2} \Sigma_{1 / 2}$ & $0.83^{a}$ & $\sim 0.1$ & 1 & 0.6 \\
${ }^{173} \mathrm{YbF}$ & $\frac{5}{2}$ & ${ }^{2} \Sigma_{1 / 2}$ & $2.1^{b}$ & 22 & 42 & 25 \\
${ }^{201} \mathrm{HgF}$ & $\frac{3}{2}$ & ${ }^{2} \Sigma_{1 / 2}$ & $4.8^{a}$ & $\sim 1$ & 10 & 6 \\
${ }^{177} \mathrm{HfF}^{+}$ & $\frac{7}{2}$ & ${ }^{3} \Delta_{1}$ & 0.5 & 20 & 33 & 20 \\
${ }^{179} \mathrm{HfF}^{+}$ & $\frac{9}{2}$ & ${ }^{3} \Delta_{1}$ & 0.5 & 14 & 26 & 16 \\
${ }^{181} \mathrm{TaN}^{1}$ & $\frac{7}{2}$ & ${ }^{3} \Delta_{1}$ & $\sim 1$ & 30 & 50 & 30 \\
${ }^{229} \mathrm{ThO}^{\frac{5}{2}}$ & ${ }^{3} \Delta_{1}$ & 1.9 & $\sim 10$ & 72 & 44 \\
${ }^{229} \mathrm{ThF}^{+}$ & $\frac{5}{2}$ & ${ }^{3} \Delta_{1}$ & 1.7 & $\sim 10$ & 65 & 39 \\
\hline \multicolumn{6}{c}{ Ref. [38]; ${ }^{b}$ Ref. [37]. }
\end{tabular}

of the quark chromo-EDMs $\left(\tilde{d}_{u}-\tilde{d}_{d}\right)$. To quantify this statement, we note that the current accuracy in measurements of the energy shift produced by the eEDM in ThO is $700 \mu \mathrm{Hz}$ [23]; it is anticipated that this may be ultimately improved by as much as $\sim 2$ orders of magnitude [47]. Similar sensitivity is anticipated in measurements based on trapped molecular ions in ${ }^{3} \Delta_{1}$ states including $\mathrm{HfF}^{+}$or $\mathrm{ThF}^{+}$22]. For comparison, for the molecule ${ }^{181} \mathrm{TaN}$ the limits on the proton EDM, $|\tilde{\theta}|$, and $\left|\tilde{d}_{u}-\tilde{d}_{d}\right|$ correspond to the shifts $\left|W_{M} M\right|<260 \mu \mathrm{Hz}, 120 \mu \mathrm{Hz}$, and $180 \mu \mathrm{Hz}$, respectively.

Comparison with TIF molecule. It is useful to compare the sensitivity to underlying sources of $\mathrm{CP}$ violation for these molecular systems with MQM contributions, to that in the diamagnetic molecule TIF. The observable T,P-odd effect in TIF is mainly produced by the nuclear SM $Q$. The SM potential for a finite nucleus has been found in Ref. 24] (unfortunately, in all molecular calculations 48 52] the authors used the finite nucleus Coulomb potential, but the Schiff moment potential remained point-like, $\left.U=-4 \pi e \frac{Q}{I}(\boldsymbol{I} \cdot \nabla \delta(r))[11,12]\right)$. In a simple valence nucleon model the SM is equal to [11, 49] $Q^{v}=\frac{d_{v}+\xi_{v} q_{v}}{10}\left[\left(t_{I}+\frac{1}{I+1}\right) r_{v}^{2}-\frac{5}{3} t_{I} r_{q}^{2}\right]$, where $r_{v}^{2}$ and $r_{q}^{2}$ are the mean squared valence nucleon and total charge distribution radii. For ${ }^{205} \mathrm{Tl}$ and ${ }^{203} \mathrm{Tl}$ nuclei the valence proton is in $3 s_{1 / 2}$ state, i.e. $I=\frac{1}{2}$ and $t_{I}=1$, and $Q^{p}=-\left(d_{v}+\right.$ $\left.\xi_{v} q_{v}\right) \frac{R}{6}$, where $R \equiv r_{v}^{2}-r_{q}^{2}$. A more accurate numerical SM calculation including the T,P-odd core polarization gives $Q^{p}=-\left[d_{p} R+\left(8.4 \eta_{p n}-7.2 \eta_{p p}\right) \cdot 10^{-21} e \cdot \mathrm{cm} \cdot \mathrm{fm}^{2}\right] / 6$, where $\eta_{p n}$ and $\eta_{p p}$ are proton-neutron and proton-proton interaction constants, $\eta_{p}=\frac{Z}{A} \eta_{p p}+\frac{N}{A} \eta_{p n}$ [25]. Different numerical nuclear calculations give $-6 \mathrm{fm}^{2}<R<5 \mathrm{fm}^{2}$ [2, 49]. In Ref. [49] the authors selected the largest of 4 results of B.A. Brown nuclear calculations $R=2.9 \mathrm{fm}^{2}$, and this value was used in all recent molecular calcu- lations 50 52 where the proton dipole moment $d_{p}$ was extracted from the TIF experiment [9].

The nuclear EDM actually gives a small but non-zero contribution to the T,P-odd frequency shift if one takes a magnetic intertaction into account [10, 48]. The valence formula for the nuclear EDM was derived in [11]: $d_{N}=\left[d_{v}-e \xi\left(q-\frac{Z}{A}\right)\right] t_{I}$. Using the molecular matrix elements calculated in ref. 52] we obtain the SM (volume) contribution $d^{V} \equiv W_{Q} Q$ and the magnetic effect contribution $d^{M}$ to the T,P-odd frequency shift in TIF:

$$
\begin{aligned}
d^{V} & =-3.4 \cdot 10^{-3} \mathrm{~Hz}\left[\frac{R}{\mathrm{fm}^{2}} \frac{10^{21} d_{p}}{e \cdot \mathrm{cm}}+8 \eta_{p n}-7 \eta_{p p}\right], \\
d^{M} & =2.0 \cdot 10^{-3} \mathrm{~Hz}\left[\frac{10^{21} d_{p}}{e \cdot \mathrm{cm}}+0.7 \eta_{p n}+0.5 \eta_{p p}\right] .
\end{aligned}
$$

Using $\eta_{p p} \approx 5 \cdot 10^{6} g\left(\bar{g}_{1}-2 \bar{g}_{2}+\bar{g}_{0}, \eta_{p n} \approx 5 \cdot 10^{6} g\left(\bar{g}_{1}+\right.\right.$ $\left.2 \bar{g}_{2}-\bar{g}_{0}\right)$, we obtain the frequency shift $\nu$ for ${ }^{205} \mathrm{TlF}$ in terms of different T,P-odd constants:

$$
\begin{aligned}
& \nu(\bar{g})=-1.0 \cdot 10^{5} \mathrm{~Hz} g\left(-0.08 \bar{g}_{1}-5.3 \bar{g}_{2}+2.6 \bar{g}_{0}\right) ; \\
& \nu(\bar{\theta})=1.0 \cdot 10^{5} \mathrm{~Hz} \bar{\theta} ; \quad \nu(\tilde{d})=2 \cdot 10^{20} \mathrm{~Hz} \frac{\left(\tilde{d}_{u}+\tilde{d}_{d}\right)}{\mathrm{cm}} .
\end{aligned}
$$

Note that the sensitivity to $\bar{g}_{0}$ and $\bar{\theta}$ is probably overestimated here since it comes from the T,P-odd core polarization, which in the case of the atomic $\mathrm{Hg}$ SM is strongly suppressed by the many-body corrections [26, 27]. These TIF results may also be used as an estimate of the SM contribution in the molecules which we considered in this paper, taking into account the scaling $Z^{2} A^{2 / 3} R_{Q}$, where $R_{Q}$ is the relativistic factor for the Schiff moment 11]. The SM contribution is 1-2 orders of magnitude smaller than the MQM contribution. The experiment with TIF [48] gave the T,P-odd frequency shift $\nu=d^{V}+d^{M}=-0.13 \pm 0.22 \mathrm{mHz}$. This gives limits $|\bar{\theta}|<4 \cdot 10^{-9}$ and $\left|\tilde{d}_{u}+\tilde{d}_{d}\right|<2 \cdot 10^{-24} \mathrm{~cm}$.

Conclusion. We find that the sensitivity to nuclear T,P-odd effects is high in paramagnetic molecules containing deformed nuclei. If measurements of EDM-like frequency shifts can be made with sensitivity an order of magnitude better than in the recent eEDM experiment using ThO molecules, then limits on several underlying parameters of hadronic T,P-violation can be improved. The molecule ${ }^{181} \mathrm{TaN}$, not considered before for EDM measurements, looks especially promising. Methods similar to those used in the ThO experiment should be applicable; even better sensitivity may be possible since the lifetime of the metastable ${ }^{3} \Delta_{1}$ state should be much longer in TaN than in ThO (due to its lower excitation energy [46, 53] ). However, further work on the molecular and nuclear structure of TaN will be needed to verify the estimates given here.

Acknowledgements. We thank L. Skripnikov for providing unpublished details of their calculation of eEDM in ThO and A. Petrov for valuable discussions. This work is supported by the Australian Research Council, the National Science Foundation, and RFBR Grant No. 14-02-00241. 
[1] D.E. Morrissey, M.J. Ramsey-Musolf, New J. Phys. 14, 125003 (2012).

[2] I.B. Khriplovich, S.K. Lamoreaux. CP violation without strangeness (Springer-Verlag, Berlin, 1997).

[3] M. Pospelov, A. Ritz, Ann. Phys. 318, 119 (2005).

[4] J. Engel, M.J. Ramsey-Musolf, U. van Kolck, Prog. Part. Nucl. Phys. 71, 21 (2013).

[5] M.D. Swallows, T.H. Loftus, W.C. Griffith, B.R. Heckel, E.N. Fortson, M.V. Romalis. Phys. Rev. A 87, 012102 (2013).

[6] M.A. Rosenberry and T.E. Chupp, Phys. Rev. Lett. 86, $22(2001)$.

[7] R.J. Holt, I. Ahmad, K. Bailey, B. Graner, J.P. Greene, W. Korsch, Z.T. Lu, P. Mueller, T.P. O'Connor, I.A. Sulai, and W.L. Trimble, Nucl. Phys. A 844, 53c (2010).

[8] E.R. Tardiff, E.T. Rand, G.C. Ball, T.E. Chupp, A.B. Garnsworthy, P. Garrett, M.E. Hayden, C.A. Kierans, W. Lorenzon, M.R. Pearson, C. Schaub, and C.E. Svensson, Hyperfine Int. 225, 197 (2014).

[9] D. Cho, K. Sangster, and E.A. Hinds, Phys. Rev. A 44, 2783 (1991).

[10] L.I. Schiff, Phys. Rev. 132, 2194 (1963).

[11] V.V. Flambaum, I.B. Khriplovich, O.P. Sushkov, Sov. Phys.-JETP 60, 873 (1984), [ZhETF, 87, 1521 (1984)].

[12] P.G.H. Sandars, Phys. Rev. Lett. 19, 1396 (1967).

[13] I.B. Khriplovich, Parity non-conservation in atomic phenomena (Gordon and Breach, New York, 1991).

[14] J.S.M. Ginges, V.V. Flambaum. Phys. Rep. 397, 63 (2004).

[15] V.V. Flambaum. Phys. Lett. B 320, 211 (1994).

[16] E.R. Meyer, J.L. Bohn, M.P. Deskevich, Phys. Rev. A 73, 062108 (2006).

[17] O.P. Sushkov, V.V. Flambaum. Zh.Eksp.Teor.Fiz. 75, 1208 (1978) [JETP 48, 608 (1978)].

[18] D.DeMille, F.Bay, S.Bickman, D.Kawall, L.Hunter, D. Krause,Jr., S.Maxwell, \& K.Ulmer in Art and Symmetry in Experimental Physics: Festschrift for Eugene D. Commins, AIP Conf.Proc.596, ed. D.Budker, P.H.Bucksbaum, and S.J.Freedman, Melville, NY (2001), p.72.

[19] N.R. Hutzler et al., Phys. Chem. Chem. Phys. 13, 18976 (2011).

[20] J.F. Barry, E.S. Shuman, and D. DeMille, Phys. Chem. Chem. Phys. 13, 18936 (2011).

[21] E. Kirilov, W.C. Campbell, J.M. Doyle, G. Gabrielse, Y.V. Gurevich, P.W. Hess, N.R. Hutzler, B.R. O'Leary, E. Petrik, B. Spaun, A.C. Vutha, D. DeMille, Phys. Rev. A 88, 013844 (2013).

[22] H. Loh, K.C. Cossel, M.C. Grau, K.-K. Ni, E.R. Meyer, J.L. Bohn, J. Ye, E.A. Cornell, Science 342, 1220 (2013).

[23] The ACME Collaboration, J. Baron, W.C. Campbell, D. DeMille, J.M. Doyle, G. Gabrielse, Y.V. Gurevich, P.W. Hess, N.R. Hutzler, E. Kirilov, I. Kozyryev, B.R. O'Leary, C.D. Panda, M.F. Parsons, E.S. Petrik, B. Spaun, A.C. Vutha, A.D. West, Science 343, 269 (2014).

[24] V.V. Flambaum, J.S.M. Ginges, Phys. Rev. A 65, 032113 (2002).

[25] V.V. Flambaum, I.B. Khriplovich, O.P. Sushkov. Nucl.
Phys. A 449, 750 (1986); Phys. Lett. B 162, 213 (1985).

[26] V.F. Dmitriev, R.A. Sen'kov, Phys. At. Nucl. 66, 1940 (2003); V.F. Dmitriev, R.A. Sen'kov, N. Auerbach, Phys. Rev. C 71, 035501 (2005).

[27] J.H. De Jesus, J. Engel, Phys. Rev. C 72, 045503 (2005); S. Ban, J. Dobaczewski, J. Engel, A. Shukla, Phys. Rev. C 82, 015501 (2010).

[28] V.F. Dmitriev, V.B. Telitsin, V.V. Flambaum, V.A. Dzuba. Phys. Rev. C 54, 3305 (1996).

[29] J.J. Hudson, D.M. Kara, I.J. Smallman, B.E. Sauer, M.R. Tarbutt, E.A. Hinds, Nature 473, 493 (2011).

[30] I.B. Khriplovich, Zh. Eksp. Teor. Fiz. 71, 51 (1976) [Sov. Phys. JETP 44, 25 (1976)].

[31] W.C. Haxton, E.M. Henley, Phys. Rev. Lett. 51, 1937 (1983).

[32] V.F. Dmitriev, I.B. Khriplovich, V.B. Telitsin, Phys. Rev. C 50, 2358 (1994).

[33] V.V. Flambaum, O.K. Vorov, Phys. Rev. C 51, 1521 (1995); Phys. Rev. C 51, 2914 (1995).

[34] R.J. Crewther, P. di Vecchia, G. Veneziano, E. Witten, Phys. Lett. B 91, 487 (1980).

[35] M. Pospelov, A. Ritz, Phys. Rev. Lett. 83, 2526 (1999).

[36] A. Bohr, B.R. Mottelson, Nuclear Structure, Vol. 2, Nuclear deformation, Ch. 5 (Benjamin, New York, 1974).

[37] M. G. Kozlov and V. F. Ezhov, Phys. Rev. A 49, 4502 (1994).

[38] M.G. Kozlov and L.N. Labzowsky, J. Phys. B 28, 1933 (1995).

[39] V.V. Flambaum, Yad. Fiz. 24,383, 1976 [Sov. J. Nucl. Phys. 24, 199 (1976)].

[40] L. Skripnikov, A.N. Petrov, and A.V. Titov, J. Chem. Phys. 139, 221103 (2013).

[41] T. Fleig and M.K. Nayak, ArXiv:1401.2284, (2014).

[42] E.R. Meyer, J.L. Bohn, and M.P. Deskevich, Phys. Rev. A 73, 062108 (2006).

[43] A.N. Petrov, N.S. Mosyagin, T.A. Isaev, and A.V. Titov, Phys. Rev. A 76, 030501 (2007).

[44] T. Fleig \& M.K. Nayak, Phys. Rev. A 88, 032514 (2013).

[45] E.R. Meyer and J.L. Bohn, Phys. Rev. A 78, 010502 (2008).

[46] R.S. Ram, J. Lièvin, and P.F. Bernath, J. Mol. Spectroscopy 215, 275 (2002).

[47] N. Hutzler, P. Hess, E. Kirilov, B. O'Leary, E. Petrik, B. Spaun, D. DeMille, G. Gabrielse, J. Doyle, Bull. Am. Phys. Soc. 57, H6.007 (2012).

[48] E.A. Hinds, P.G.H. Sandars, Phys. Rev. A 21, 471 (1980).

[49] P.V. Coveney, P.G.H. Sandars, J. Phys. B 16, 471 (1983).

[50] F.A. Parpia, J. Phys. B 30, 3983 (1997).

[51] H.M. Quiney, J.K. Laerdahl, K. Faegri, Jr., and T. Saue, Phys. Rev. A 57, 920 (1998).

[52] A.N. Petrov, N.S. Mosyagin, T.A. Isaev, A.V. Titov, V.F. Ezhov, E. Eliav, U. Kaldor, Phys. Rev. Lett. 88, 073001 (2002).

[53] A.C. Vutha, W.C. Campbell, Y.V. Gurevich, N.R. Hutzler, M. Parsons, D. Patterson, E. Petrik, B. Spaun, J.M. Doyle, G. Gabrielse, and D. DeMille, J. Phys. B 43, 074007 (2010). 